\title{
Clinical outcomes and risk factors for death from disseminated histoplasmosis in patients with AIDS who visited a high-complexity hospital in Campo Grande, MS, Brazil
}

\author{
Barbara Cristina Scarcelli Boigues ${ }^{[1]}$, Anamaria Mello Miranda Paniago ${ }^{[1]}$, \\ Gláucia Moreira Espíndola Lima ${ }^{[2]}$, Maina de Oliveira Nunes ${ }^{[2]}$ and \\ Silvia Naomi de Oliveira Uehara ${ }^{[1]}$
}

[1]. Divisão de Doenças Infecciosas, Universidade Federal de Mato Grosso do Sul, Campo Grande, MS, Brasil.

[2]. Laboratório de Micologia Médica, Universidade Federal de Mato Grosso do Sul, Campo Grande, MS, Brasil.

\begin{abstract}
Introduction: Disseminated histoplasmosis (DH) is a systemic mycosis caused by Histoplasma capsulatum (H. capsulatum) and is characterized by progressive and fatal evolution in immunocompromised patients. Moreover, it is considered an AIDSdefining disease. Methods: We performed an observational, analytical, retrospective study to identify the clinical outcomes and risk factors for death from DH in patients with AIDS at an infectious diseases service facility in Brazil between September 2011 and July 2016. Patients with a positive serology for HIV and DH were diagnosed via direct examination and/or positive cultures for H. capsulatum. Results: Twenty-three patients were included in this study. Approximately, $82.6 \%$ were men, with a mean age of $41.0 \pm 11.5$ years, and $52.2 \%$ had a concomitant diagnosis of AIDS and DH. The median CD4+ T cell count was 19 cells/ $\mathrm{mm}^{3}$, and $56.5 \%$ of the patients died. The most frequently observed symptoms were fever, dyspnea, and skin lesions. On the basis of a comparative analysis of those who died and survived, the absence of splenomegaly and hepatomegaly and the presence of $H$. capsulatum in the peripheral blood were considered as risk factors for death. Those who died had a higher leukocyte count; CRP, urea, and lactate dehydrogenase levels; AST index; and international normalized ratio prothrombin time. The serum total protein and albumin levels of the patients were lower. Conclusions: The mortality rate for DH is high among severely immunocompromised patients with AIDS. The risk factors for death were those traditionally associated with blood dyscrasia, inflammatory activity, as well as increased renal and nutritional impairment.
\end{abstract}

Keywords: Histoplasmosis. Histoplasma capsulatum. AIDS.

\section{INTRODUCTION}

Histoplasmosis is a systemic mycosis caused by Histoplasma capsulatum (H. capsulatum), and it can manifest in several forms: asymptomatic, acute pulmonary, chronic pulmonary, or disseminated. Disseminated histoplasmosis (DH) is characterized by hematogenous dissemination with hemolymphopoietic organ involvement. This phenomenon mainly occurs because of reactivation of a latent infection or through significant exogenous exposure, with progressive evolution and high mortality found in immunocompromised patients. DH is considered an opportunistic infection that occurs in $2-8 \%$ of patients with acquired immunodeficiency syndrome (AIDS),

Corresponding author: Barbara Cristina Scarcelli Boigues e-mail: bboigues@hotmail.com; barbaraboigues@hotmail.com Received 11 October 2017

Accepted 20 March 2018 usually when the cluster of differentiation $4+(\mathrm{CD} 4+) \mathrm{T}$ cell count is $<150$ cells $/ \mathrm{mm}^{3}$. Moreover, DH is more likely to develop in the absence of antiretroviral therapy (ART) use $\mathrm{u}^{1-4}$.

The mortality rates are as high as $20-70 \% \%^{5-9}$. Because of these poor outcomes, the identification of the prognostic factors may help determine patients who require more aggressive treatment.

There are only few studies on the outcomes of patients with concomitant diagnosis of AIDS and DH. The risk factors for death from DH in patients with AIDS include a platelet count $<100,000$ platelets $/ \mathrm{mm}^{3}$, fungemia, anemia, elevated aspartate aminotransferase (AST) and urea levels, acute renal failure, and respiratory insufficiency ${ }^{3,6,7,10}$.

At the Hospital Universitário Maria Aparecida Pedrossian/ Universidade Federal do Mato Grosso do Sul (HUMAP/ UFMS), which serves as a reference center at a tertiary level hospital for parasitic and infectious diseases, the latest survey on DH was conducted between 1998 and 2005, with no new data obtained after the implementation and increased availability 
of highly active antiretroviral therapy (HAART) ${ }^{11}$. These findings emphasize the need to assess this issue. Therefore, this study aimed to describe the clinical outcomes of patients with a concomitant diagnosis of AIDS and DH. Moreover, the associated risk factors that can lead to death were examined.

\section{METHODS}

This is an observational, analytical, retrospective study that used the medical and mycology laboratory records of patients admitted to the HUMAP/UFMS infectious disease department between September 2011 and July 2016.

Individuals over the age of 18 years were included in this study. Furthermore, individuals were included in this investigation if they had positive serological findings for HIV, detectable human immunodeficiency virus-ribonucleic acid (HIV-RNA) levels at least once during their lifetime, and a positive culture and/or a positive direct mycological examination for $H$. capsulatum using different specimens. With regard to viral load, it could either be detectable/quantifiable or undetectable during the study period. The samples were collected at the time of histoplasmosis investigation and diagnosis.

We selected the medical records of 23 patients and developed a systemized questionnaire that aimed to collect their epidemiological, clinical and laboratory data during hospitalization. Organic samples, such as bone marrow $(\mathrm{n}=12)$, blood $(\mathrm{n}=7)$, tracheal secretions $(\mathrm{n}=1)$, and skin fragments $(\mathrm{n}=6)$, were submitted for direct microscopy (stained by Giemsa) and/ or for cultures to isolate H. capsulatum in Mycosel Agar and Sabouraud Dextrose Agar with Chloramphenicol for 60 days.

Splenomegaly and hepatomegaly were evaluated via physical examination.

Outcome data (dead or alive) within 30 days from the start of follow-up were also obtained from the medical records, and patients who were categorized as dead were those who died due to histoplasmosis during follow-up at the service facility.

Induction therapy comprised $0.7-1 \mathrm{mg} / \mathrm{kg} /$ day (maximum of $50 \mathrm{mg} /$ day) amphotericin B deoxycholate (AmBd) for 2 weeks or $3 \mathrm{mg} / \mathrm{kg}$ /day amphotericin B (LFAmB) lipid formulations for 2 weeks if the patient had initial signs of renal impairment. The consolidation treatment was initiated with $200 \mathrm{mg}$ itraconazole twice a day for at least 12 months.

Data were analyzed with the Statistical Package for the Social Sciences (SPSS) version 22 (IBM Corporation, Armonk, NY, USA).

Categorical variables were presented in percentages. For comparative purposes, either the chi-squared test or Fisher's exact test (for small samples) was used. Continuous variables were expressed as means or medians according to the data's normal or non-normal distribution. Student's t-test and the Mann-Whitney U test were performed for comparisons. The Shapiro-Wilk test was used to verify a normal distribution in the sample. Due to the small sample size, logistic regression analysis cannot be performed to evaluate the factors that were independently associated with patient outcomes (dead or alive).

\section{Ethical considerations}

This study was approved by the human research ethics committee of the Universidade Federal de Mato Grosso do Sul (approval number: 73178017.8.0000.0021). This study was in accordance with the Declaration of Helsinki.

\section{RESULTS}

In this study, patients were followed-up during the hospitalization period, and the median time between diagnosis and the last clinical evaluation while patients were hospitalized was 6 days $[2,20]$. Among those who died, the median time was 4 days $[1,17]$. In total, 23 patients had a diagnosis of $\mathrm{DH}$, which was confirmed via direct examination $(1 / 23,4.3 \%)$ and/or culture $(22 / 23,95.7 \%)$. Of these patients, $82.6 \%$ were men with a mean age of $41.0 \pm 11.5$ years. Moreover, $65.2 \%$ were non-white, $52.2 \%$ were born in Mato Grosso do Sul, and all the patients were from Brazil. Approximately 65.2\% were from Campo Grande City. Furthermore, $95.7 \%$ of the patients experienced their first histoplasmosis episode, and $56.5 \%$ of these individuals died.

Twelve (52.2\%) patients had a concomitant diagnosis of AIDS and DH. The median time between the diagnosis of HIV and histoplasmosis was 10 weeks $[0,706]$. The mean time between symptom onset and the diagnosis of histoplasmosis was $6.7 \pm 5.6$ weeks. Moreover, the median LTCD4+ $(\mathrm{CD} 4+\mathrm{T}$ cell- lymphocytes) count at or near the time of hospital admission was 19 cells $/ \mathrm{mm}^{3}[1,589]$. Finally, the most frequently observed symptoms were fever, dyspnea, skin lesions, and cough (Table 1).

Hypertension and diabetes mellitus were observed in 8.7\% and $4.3 \%$ of the patients, respectively (Table 2 ). The most

TABLE 1: Frequency (percentage and number) of symptoms among patients with $\mathrm{DH}$.

\begin{tabular}{lc}
\hline Symptom & Frequency \\
\cline { 2 - 2 } & $\%(\mathbf{n})$ \\
\hline Fever & $87.0(20)$ \\
Dyspnea & $60.9(14)$ \\
Cough/skin lesions* & $52.2(12)$ \\
Bleeding/diarrhea/hepatomegaly* & $43.5(10)$ \\
Anorexia & $39.1(9)$ \\
Fatigue & $30.4(7)$ \\
Vomiting & $21.7(5)$ \\
Splenomegaly/dysphagia* & $17.4(4)$ \\
Reduced consciousness/chest pain/jaundice* & $13.0(3)$ \\
Chills/lesions in the oral mucosa/nausea* & $4.3(1)$ \\
\hline
\end{tabular}

$\mathrm{DH}$ : disseminated histoplasmosis; \%: percentage; $\mathbf{n}$ : number. * $\mathrm{n}$ is described for each symptom. 
TABLE 2: Distribution of patients with DH according to demographic, clinical, and therapeutic characteristics as well as comorbidity and mortality.

\begin{tabular}{|c|c|c|c|c|c|c|}
\hline \multirow[t]{2}{*}{ Variables } & \multirow[b]{2}{*}{ Total } & \multicolumn{2}{|c|}{ Patients, \% (n) } & \multirow[b]{2}{*}{ OR } & \multirow[b]{2}{*}{$95 \% \mathrm{Cl}$} & \multirow[b]{2}{*}{ p-value } \\
\hline & & dead (13) & alive (10) & & & \\
\hline Sex & 23 & & & & & \\
\hline male & & $52.6(10)$ & $47.4(9)$ & 1 & & 0.604 \\
\hline female & & $75.0(3)$ & $25.0(1)$ & 2.70 & $(0.24-30.84)$ & \\
\hline Skin color & 23 & & & & & \\
\hline non-white & & $66.7(10)$ & $33.3(5)$ & 1 & & 0.221 \\
\hline White & & $37.5(3)$ & $62.5(5)$ & 0.30 & $(0.50-1.80)$ & \\
\hline Smoker ${ }^{a}$ & 13 & & & & & \\
\hline no & & $50.0(4)$ & $50.0(4)$ & 1 & & 1 \\
\hline yes & & $60.0(3)$ & $40.0(2)$ & 1.50 & $(0.16-14.42)$ & \\
\hline Viral hepatitis & 23 & & & & & \\
\hline yes & & $0.0(0)$ & $100.0(1)$ & 1 & & 0.435 \\
\hline no & & $59.1(13)$ & $40.9(9)$ & - & - & \\
\hline VL & 23 & & & & & \\
\hline yes & & $0.0(0)$ & $100.0(2)$ & 1 & & 0.178 \\
\hline no & & $61.9(13)$ & $38.1(8)$ & - & - & \\
\hline DM & 23 & & & & & \\
\hline yes & & $100.0(1)$ & $0.0(0)$ & 1 & & 1.00 \\
\hline no & & $54.5(12)$ & $45.5(10)$ & - & - & \\
\hline SAH & 23 & & & & & \\
\hline yes & & $100.0(2)$ & $0.0(0)$ & 1 & & 0.486 \\
\hline no & & $52.4(11)$ & $47.6(10)$ & - & - & \\
\hline Cryptococcosis & 23 & & & & & \\
\hline yes & & $100.0(2)$ & $0.0(0)$ & 1 & & 0.486 \\
\hline no & & $52.4(11)$ & $47.6(10)$ & - & - & \\
\hline NTX & 23 & & & & & \\
\hline yes & & $25(1)$ & $75.0(3)$ & 1 & & 0.281 \\
\hline no & & $63.2(12)$ & $36.8(7)$ & 5.14 & $(0.45-59.46)$ & \\
\hline Pneumocystosis & 23 & & & & & \\
\hline yes & & $83.3(5)$ & 16. 7 (1) & 1 & & 0.179 \\
\hline no & & $47.1(8)$ & $52.9(9)$ & 0.18 & $(0.02-1.86)$ & \\
\hline BP & 23 & & & & & \\
\hline yes & & $100.0(4)$ & $0.0(0)$ & 1 & & 0.104 \\
\hline no & & $47.4(9)$ & $52.6(10)$ & - & - & \\
\hline PT & 23 & & & & & \\
\hline yes & & $33.3(2)$ & $66.7(4)$ & 1 & & 0.341 \\
\hline no & & $64.7(11)$ & $35.3(6)$ & 3.67 & $(0.51-26.22)$ & \\
\hline Use of HAART & 23 & & & & & \\
\hline yes & & $40.0(4)$ & $60.0(6)$ & 1 & & 0.222 \\
\hline no & & $62.2(9)$ & $30.8(4)$ & 3.38 & $(0.60-19.01)$ & \\
\hline Weight loss & 23 & & & & & \\
\hline yes & & $53.3(8)$ & $47.7(7)$ & 1 & & 1.00 \\
\hline no & & $62.5(5)$ & $37.5(3)$ & 1.46 & $(0.25-8.43)$ & \\
\hline
\end{tabular}


TABLE 2: Continuation.

\begin{tabular}{|c|c|c|c|c|c|c|}
\hline \multirow[t]{2}{*}{ Variables } & \multirow[b]{2}{*}{ Total } & \multicolumn{2}{|c|}{ Patients, \% (n) } & \multirow[b]{2}{*}{ OR } & \multirow[b]{2}{*}{$95 \% \mathrm{Cl}$} & \multirow[b]{2}{*}{$\mathrm{p}$-value } \\
\hline & & dead (13) & alive (10) & & & \\
\hline yes & & $50.0(10)$ & $50.0(10)$ & 1 & & 0.229 \\
\hline no & & $100.0(3)$ & $0.0(0)$ & - & - & \\
\hline yes & & $41.7(5)$ & $58.3(7)$ & 1 & & 0.214 \\
\hline no & & $72.7(8)$ & $27.3(3)$ & 3.73 & $(0.65-21.58)$ & \\
\hline Dyspnea & 23 & & & & & \\
\hline yes & & $71.4(10)$ & $28.6(4)$ & 1 & & 0.102 \\
\hline no & & $68.4(13)$ & $31.6(6)$ & - & - & \\
\hline Hepatomegaly & 23 & & & & & \\
\hline yes & & $30.0(3)$ & $70.0(7)$ & 1 & & 0.04 \\
\hline no & & $76.9(10)$ & $23.1(3)$ & 7.78 & $(1.20-50.42)$ & \\
\hline Skin lesions & 23 & & & & & \\
\hline yes & & $41.7(5)$ & $58.3(7)$ & 1 & & 0.214 \\
\hline no & & $72.7(8)$ & $27.3(3)$ & 3.73 & $(0.65-21.57)$ & \\
\hline Bleeding & 23 & & & & & \\
\hline yes & & $100.0(2)$ & $0.0(0)$ & 1 & & 0.486 \\
\hline no & & $52.4(11)$ & $47.6(10)$ & - & - & \\
\hline Neurological symptoms ${ }^{b}$ & 23 & & & & & \\
\hline yes & & $66.7(2)$ & $33.3(1)$ & 1 & & 1.00 \\
\hline no & & $55.0(11)$ & $45.0(9)$ & 0.61 & $(0.05-7.88)$ & \\
\hline Histoplasm (hemogram) ${ }^{c}$ & 23 & & & & & \\
\hline no & & $37.5(6)$ & $62.5(10)$ & 1 & & 0.007 \\
\hline yes & & $100.0(7)$ & $0.0(0)$ & - & - & \\
\hline Induction treatment $^{d}$ & 23 & & & & & \\
\hline yes & & $52.4(11)$ & $47.6(10)$ & 1 & & 0.486 \\
\hline no & & $100.0(2)$ & $0.0(0)$ & - & - & \\
\hline Consolidation treatment $\mathrm{e}^{\mathrm{e}}$ & 23 & & & & & \\
\hline yes & & $0.0(0)$ & $100.0(3)$ & 1 & & 0.068 \\
\hline no & & $65.0(13)$ & $35.0(7)$ & - & - & \\
\hline
\end{tabular}

DH: disseminated histoplasmosis; OR: odds ratio; 95\% Cl: confidence intervals 95\%; VL: visceral leishmaniasis; DM: diabetes mellitus; SAH: systemic arterial hypertension; NTX: neurotoxoplasmosis; BP: bacterial pneumonia; PT: pulmonary tuberculosis; HAART: highly active antiretroviral therapy.

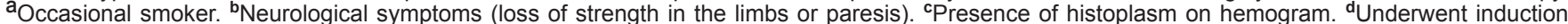

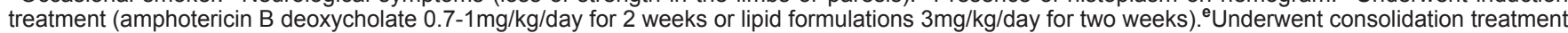
(itraconazole $400 \mathrm{mg} /$ day for at least 12 months). 
frequently associated infectious diseases were pneumocystosis and tuberculosis (observed in $26 \%$ of patients), followed by neurotoxoplasmosis and bacterial pneumonia $(17.4 \%)$, visceral leishmaniasis and cryptococcosis (8.7\%), and syphilis (4.3\%) (Table 2). Of these, two patients were diagnosed with pulmonary tuberculosis based on sputum culture, and two different patients had cryptococcosis according to the histopathological findings in one patient and blood culture in another. Other associated diseases had a presumptive diagnosis based on positive therapeutic responses.

Of the 23 patients included in our study, $91.3 \%$ had an initial treatment with amphotericin B (19 patients with AmBd and two patients with LFAmB), and two patients died before treatment initiation. However, only $13 \%$ underwent consolidation treatment and none underwent maintenance treatment (Table 2). The median AmBd accumulated dose was $300 \mathrm{mg}$ $[30,920]$. One patient used liposomal $\mathrm{AmB} 250 \mathrm{mg}$ and the other used 1,100mg of lipid complex AmBd. Non-treatment compliance was attributed to premature death and the loss of follow-up during the outpatient period.

Among the 23 patients included in this investigation, $56.5 \%$ died due to histoplasmosis complications within 30 days from the start of follow-up. The comparative analysis between the dead and alive groups emphasized that the absence of splenomegaly and hepatomegaly and the presence of H. capsulatum in the peripheral blood were the risk factors for death (Table 2).

Among those who died, higher leukocyte count and C-reactive protein, creatinine, urea, prothrombin time, lactic dehydrogenase, and AST levels (AST observed value/upper limit of AST reference level) were observed. In addition, those who died had lower total serum protein and albumin levels, and the time interval between DH diagnosis and outcome was also lower $(p=0.012)$ (Table 3). No significant difference was observed in terms of the median LTCD4+ count between those who died and survived.

\section{DISCUSSION}

In this study, 23 cases of DH and AIDS were observed in the study population over a period of 4 years and 10 months. These cases were found from the laboratory records because the total number of hospitalized patients with AIDS could not be identified owing to the hospitalizations that occurred in different divisions of the hospital. Therefore, the prevalence of $\mathrm{DH}$ and AIDS coinfection in patients during the study period was not evaluated because the number of cases might have varied in the different population at risk. However, the number of cases found was $53 \%$ higher than expected when compared with the previous study in our institution that obtained data from January 1998 to December 2005. However, no statistically significant difference was observed on using the same diagnostic methods applied in our study ${ }^{11}$.

The HUMAP/UFMS mycology laboratory does not use blood lysis/centrifugation techniques before placing the samples on Sabouraud Agar. This practice maybe a possible explanation in addition to other reasons as to why DH is less detected or identified at a later time because this procedure more likely accelerates the detection of the fungus in a non-automated culture.

In addition, it is worth noting that the higher prevalence of this infection in men and young adults was similar to the findings of other studies and may reflect the predominance of AIDS among men in all Brazilian regions ${ }^{3,11,12}$.

Despite the increased availability of diagnostic resources, there may be no repercussions for the early detection of HIV infection ${ }^{3,11}$. The LTCD4+ count was measured in $65.2 \%$ $(\mathrm{n}=15)$ of patients (median: 19 cells $\left./ \mathrm{mm}^{3}\right)$, indicating severe immunosuppression in the dead and alive groups. This finding was lower than the cutoff value adopted by Pontes ${ }^{3}$.

Most (52.2\%) of the patients had a concomitant diagnosis of DH and AIDS, and 56.5\% died from DH, suggesting a high mortality rate within 30 days from the start of follow-up. Fever and bleeding that were evident among individuals in our study reflected the systemic nature of the disease. The presence of dyspnea and cough in more than half of the patients was also emphasized. The possible association of these symptoms with other opportunistic diseases affecting the respiratory system could not be ruled out because radiological and laboratory findings that overlapped with pneumocystosis, tuberculosis, histoplasmosis, and bacterial pneumonia made it difficult to obtain a differential diagnosis ${ }^{3,11}$.

The long interval between the onset of histoplasmosis symptoms and the disease diagnosis may have contributed to the severity of the cases, although there was no significant difference between the dead and alive groups because both groups were severely immunocompromised.

The mortality rate that was maintained when compared with that of a study carried out between 1998 and 2005 in the same service facility $(\mathrm{p}=0.555)$ was significantly high, despite the evolution and increased availability of HAART ${ }^{11}$. When examining the few cases in which the LTCD4+ count was high, the last available count may have been obtained before admission or withdrawal from HAART, and no new data were collected when patients died early.

The mortality rate in our study was higher than that observed by Gutierrez et al. in Panama (56.5\% versus $12.5 \%$, respectively; $\mathrm{p}<0.005)$, Pontes et al. in Brazil (56.5\% versus $30.59 \%$, respectively; $\mathrm{p}<0.05$ ), Peigne et al. in France (56.5\% versus $22.0 \%$, respectively; $\mathrm{p}<0.05$ ), and Couppié et al. in French Guiana (56.5\% versus $22.0 \%$, respectively; $p<0.05)^{3,5,6,13}$. However, the mortality rate was similar to that observed by Baddley et al. in the USA (56.5\% versus $39.1 \%$ respectively; $\mathrm{p}>0.05$ ) and Samayoa et al. in Guatemala ${ }^{7,8}$.

Of note, the studies differed in the time to death after DH diagnosis. Studies that considered death within 30 days had lower mortality rates ${ }^{3,6}$. They also differed in terms of the selection of cases, and those that included cases confirmed via histopathological examination had a lower mortality rate r.,13 $^{5}$ . In our study, microbiology laboratory records were used to search for the cases. Thus, only cases with positive culture and/ or mycological direct were included. This process resulted in a 
TABLE 3: Statistical comparison of age, laboratory results, and disease duration of patients with $\mathrm{DH}$ according to their baseline characteristics ( $\mathrm{n}=23$ ).

\begin{tabular}{|c|c|c|c|}
\hline Patients with DH & Dead (13) & Alive (10) & $\mathrm{p}$-value \\
\hline Age upon admission (years) & $44.00 \pm 13.04(36.12-51.88)$ & $37.20 \pm 8.39(31.20-43.20)$ & $0.116^{*}$ \\
\hline $\begin{array}{l}\text { Time interval between symptoms and } \\
\text { histoplasmosis diagnosis (weeks) }\end{array}$ & $6.38 \pm 6.01(2.75-10.01)$ & $7.10 \pm 5.34(3.28-10.92)$ & $0.77^{*}$ \\
\hline Reported weight loss (kg) & $14.00 \pm 3.08(10.17-17.83)$ & $9.67 \pm 3.51(0.94-18.39)$ & $0.116^{*}$ \\
\hline Hematocrit (\%) & $27.09 \pm 6.55(23.13-31.04)$ & $25.18 \pm 4.65(21.85-28.51)$ & $0.445^{*}$ \\
\hline CRP level (mg/dL) & $189.90 \pm 119.84(117.48-262.32)$ & $102.05 \pm 71.92(50.60-153.50)$ & $0.041^{*}$ \\
\hline Total proteins level $(\mathrm{g} / \mathrm{dL})^{\mathrm{b}}$ & $5.23 \pm 1.07(4.59-5.88)$ & $6.34 \pm 1.24(5.45-7.23)$ & $0.032^{*}$ \\
\hline $\mathrm{PTT} / \mathrm{INR}^{\mathrm{d}}$ & $1.56 \pm 0.39(1.28-1.83)$ & $1.76 \pm 0.67(0.69-2.82)$ & $0.492^{*}$ \\
\hline AST index & $6.96 \pm 5.19(3.83-10.10)$ & $3.50 \pm 2.75(1.53-5.46)$ & $0.053^{*}$ \\
\hline ALT index & $1.69 \pm 0.93(1.13-2.25)$ & $1.23 \pm 0.89(0.59-1.87)$ & $0.246^{*}$ \\
\hline \multicolumn{4}{|l|}{ Variables [median (min, max)] } \\
\hline $\begin{array}{l}\text { Time interval between diagnosis of HIV and } \\
\text { histoplasmosis (weeks) }^{\mathrm{e}}\end{array}$ & $4.00[0,706]$ & $14.00[0,468]$ & $0.651^{* *}$ \\
\hline $\begin{array}{l}\text { Time interval between histoplasmosis diagnosis and } \\
\text { outcomes (days) }^{f}\end{array}$ & $4[1,17]$ & $16.5[7,51]$ & $0.012^{* *}$ \\
\hline HIV viral load closest to HIV diagnosis(copies $/ \mathrm{mL})^{j}$ & $103246.25[460,395732]$ & $245124.00[63,1140294]$ & $0.541^{* *}$ \\
\hline Leukocyte level $\left(\mathrm{mm}^{3}\right)$ & $8410.00[2860,27500]$ & $3156.00[1450,6330]$ & $0.003^{* *}$ \\
\hline Platelet count $\left(\mathrm{mm}^{3}\right)$ & $149692.31[14000,377000]$ & $89200.00[19000,201000]$ & $0.284^{* *}$ \\
\hline $\mathrm{ESR}(\mathrm{mm} / \mathrm{h})$ & $17.43[9,25]$ & $38.00[9,91]$ & $0.097^{* *}$ \\
\hline Globulin level (g/dL) & $3.30[2.50,4.80]$ & $3.96[2.40,6.20]$ & $0.115^{* *}$ \\
\hline Creatinine level (mg/dL) & $3.09[0.60,15.0]$ & $0.83[0.30,1.50]$ & $0.018^{* *}$ \\
\hline Urea level (mg/dL) & $80.86[18.10,256]$ & $35.42[12.10,63]$ & $0.03^{* *}$ \\
\hline Potassium level (mmol/L) & $4.28[2.2,7.4]$ & $4.10[3.4,4.9]$ & $0.539 * *$ \\
\hline LDH level (U/L) & $2728.75[593,5854]$ & $617.25[146,1733]$ & $0.000^{* *}$ \\
\hline Amphotericin B (mg) & $120[30,1100]$ & $520[90,920]$ & $0.086^{* *}$ \\
\hline
\end{tabular}

DH: disseminated histoplasmosis; SD: standard deviation;95\% Cl: confidence intervals 95\%; CRP: C-reactive protein; PT: prothrombin time; INR: international normalized ratio; PTT: activated partial thromboplastin time; AST: aspartate aminotransferase; ALT: alanine aminotransferase; HIV: human immunodeficiency virus; CD4+: cluster of differentiation 4 (CD4); ESR: erythrocyte sedimentation rate; LDH: lactate dehydrogenase; U/L: units per liter. ${ }^{a}$ Time interval between the onset of symptoms and the diagnosis of histoplasmosis (weeks). ${ }^{\mathbf{b}}$ Total proteins. ${ }^{\mathrm{c}}$ Prothrombin activation time. ${ }^{\mathrm{d}}$ Activated partial thromboplastin time. ${ }^{\mathrm{e}}$ Time interval between diagnosis of HIV and diagnosis of histoplasmosis (weeks). ${ }^{\mathrm{f}} \mathrm{Time}$ interval between the diagnosis of histoplasmosis and outcome (days). ${ }^{\mathrm{g}} \mathrm{CD} 4+\mathrm{T}$ cell count closest to hospitalization. ${ }^{\mathrm{h}} \mathrm{CD} 4+\mathrm{T}$ cell count closest to HIV diagnosis. ${ }^{\mathrm{i}} \mathrm{HIV}$ viral load closest to hospitalization. 'HIV viral load closest to HIV diagnosis. *Student's t-test. **Mann-Whitney U test. 
sample of more severe diseases and, therefore, higher lethality rate. The fact that $7 / 23$ patients had $H$. capsulatum in the peripheral blood reinforces the severity of DH in these cases.

The perception that the visualization of $H$. capsulatum in the peripheral blood would indicate greater disease severity was confirmed by the significant difference in its occurrence among patients who eventually died.

The absence of hepatomegaly or splenomegaly was more frequent among those who died. This finding could be explained by the fact that patients with hepatomegaly or splenomegaly are examined earlier for DH. However, the interval between the onset of symptoms and the time of diagnosis was not different among patients with and without hepatomegaly or splenomegaly.

Several laboratory markers suggesting increased disease severity, such as elevated $C$-reactive protein (CRP), lactate dehydrogenase, and leukocyte levels, low albumin level, increased nitrogen compounds, and the presence of hepatocellular lesions, were significantly different among those who died. Some of these factors were also observed by different authors ${ }^{3,6,7}$. Although patients with $\mathrm{DH}$ also have elevated leukocyte levels in the peripheral blood and C-reactive protein levels in the serum, these alterations are also frequent in secondary bacterial infections that can occur concomitantly with $\mathrm{DH}$ and worsen its prognosis.

In contrast to other studies, the platelet count was not different between the groups ${ }^{6}$. We did not observe a lower CD4 count among those who died, which is similar to the findings of other studies 7 . Of note, the CD4+ count test was performed in only 15 patients, which is an insufficient number for a satisfactory statistical analysis.

This study has some limitations. The sample size of the patients was small, which limits multivariable analysis. We also assumed that there was an association between tuberculosis and/ or pneumocystosis based on clinical and radiological findings and therapeutic responses to broad antibacterial and antifungal agents.

The review of the patient medical records emphasized numerous issues that are inherently associated with retrospective studies, such as the lack of socio-epidemiological data. Moreover, the lack of adherence to outpatient follow-up and post-discharge maintenance treatment indicates the need to both obtain additional information and to call absent patients for follow-up. Furthermore, clinicians must ensure that timely follow-up appointments are made at outpatient infectious disease clinics.

In conclusion, the mortality rate for $\mathrm{DH}$ was high among severely immunocompromised patients with AIDS. The risk factors for death were those traditionally associated with blood dyscrasia, inflammatory activity, as well as renal and nutritional impairment.

\section{Acknowledgments}

We would like to thank Everton Lemos and Sandra Maria do Valle Leone de Oliveira for their support in formatting the article and submitting it to the ethics committee.

\section{Conflict of interest}

The authors declare that there is no conflict of interest.

\section{Financial support}

This study was funded by the researcher.

\section{REFERENCES}

1. Kauffman CA. Histoplasmosis: a clinical and laboratory update. Clin Microbiol Rev. 2007;20(1):115-32.

2. Focaccia R, Veronesi R. Tratado de Infectologia. 2 Volumes. $5^{\text {a }}$ edição. São Paulo: Editora Atheneu; 2015.

3. Pontes LB, Leitão TMJS, Lima GG, Gerhard ES, Fernandes TA. Características clínico-evolutivas de 134 pacientes com histoplasmose disseminada associada a SIDA no Estado do Ceará. Rev Soc Bras Med Trop. 2010;43(1):27-31.

4. Ministério da saúde (MS). Secretaria de vigilância em saúde. Departamento de DST Aids e Hepatites virais. Protocolo clínico e diretrizes terapêuticas para manejo da infecção pelo HIV em adultos. Brasília: MS; 2013.

5. Peigne V, Dromer F, Elie C, Lidove O, Lortholary O, French Mycosis Study G. Imported acquired immunodeficiency syndrome-related histoplasmosis in metropolitan France: a comparison of pre-highly active anti-retroviral therapy and highly active anti-retroviral therapy eras. Am J Trop Med Hyg. 2011;85(5):934-41.

6. Couppie P, Sobesky M, Aznar C, Bichat S, Clyti E, Bissuel F. Histoplasmosis and acquired immunodeficiency syndrome: a study of prognostic factors. Clin Infect Dis. 2004;38(1):134-8.

7. Baddley JW, Sankara IR, Rodriquez JM, Pappas PG, Many Jr WJ. Histoplasmosis in HIV-infected patients in a southern regional medical center: poor prognosis in the era of highly active antiretroviral therapy. Diagn Microbiol Infect Dis. 2008;62(2): 151-6.

8. Samayoa B, Roy M, Cleveland AA, Medina N, Lau-Bonilla D, Scheel CM. High mortality and coinfection in a prospective cohort of human immunodeficiency virus/acquired immune deficiency syndrome patients with histoplasmosis in Guatemala. Am J Trop Med Hyg. 2017;97(1):42-48.

9. Nacher M, Adenis A, Blanchet D, Vantilcke V, Demar M, Basurko C. Risk factors for disseminated histoplasmosis in a cohort of HIV-infected patients in French Guiana. PLoS Negl Trop Dis. 2014;8(1):e2638.

10. Daher EF, Silva Jr GB, Barros FA, Takeda CF, Mota RM, Ferreira MT, et al. Clinical and laboratory features of disseminated histoplasmosis in HIV patients from Brazil. Trop Med Int Health. 2007;12(9):1108-15.

11. Chang MR, Taira CL, Paniago AM, Taira DL, Cunha RV, Wanke B. Study of 30 cases of histoplasmosis observed in the Mato Grosso do Sul State, Brazil. Rev Inst Med Trop Sao Paulo. 2007;49(1):37-9.

12. Ministério da Saúde. Secretaria de Vigilância em Saúde. Departamento de DST Aids e Hepatites Virais. Boletim Epidemiológico HIV/AIDS. Brasília: MS; 2016.

13. Gutierrez ME, Canton A, Sosa N, Puga E, Talavera L. Disseminated histoplasmosis in patients with AIDS in Panama: a review of 104 cases. Clin Infect Dis. 2005;40(8):1199-202. 